\title{
Anti-tetherin activities in Vpu-expressing primate lentiviruses
}

\author{
Su Jung Yang, Lisa A Lopez, Heiko Hauser, Colin M Exline, Kevin G Haworth, Paula M Cannon*
}

\begin{abstract}
Background: The anti-viral activity of the cellular restriction factor, BST-2/tetherin, was first observed as an ability to block the release of Vpu-minus HIV-1 from the surface of infected cells. However, tetherin restriction is also counteracted by primate lentiviruses that do not express a Vpu protein, where anti-tetherin functions are provided by either the Env protein (HIV-2, SIVtan) or the Nef protein (SIVsm/mac and SIVagm). Within the primate lentiviruses, Vpu is also present in the genomes of SIVcpz and certain SIVsyk viruses. We asked whether, in these viruses, anti-tetherin activity was always a property of $\mathrm{Vpu}$, or if it had selectively evolved in HIV-1 to perform this function.

Results: We found that despite the close relatedness of HIV-1 and SIVcpz, the chimpanzee viruses use Nef instead of Vpu to counteract tetherin. Furthermore, SIVcpz Nef proteins had activity against chimpanzee but not human tetherin. This specificity mapped to a short sequence that is present in the cytoplasmic tail of primate but not human tetherins, and this also accounts for the specificity of SIVsm/mac Nef for primate but not human tetherins. In contrast, Vpu proteins from four diverse members of the SIVsyk lineage all displayed an anti-tetherin activity that was active against macaque tetherin. Interestingly, Vpu from a SIVgsn isolate was also found to have activity against human tetherin.
\end{abstract}

Conclusions: Primate lentiviruses show a high degree of flexibility in their use of anti-tetherin factors, indicating a strong selective pressure to counteract tetherin restriction. The identification of an activity against human tetherin in SIVgsn Vpu suggests that the presence of Vpu in the ancestral SIVmus/mon/gsn virus believed to have contributed the $3^{\prime}$ half of the HIV-1 genome may have played a role in the evolution of viruses that could counteract human tetherin and infect humans.

\section{Background}

The release of HIV-1 and other enveloped viruses from the surface of infected cells is reduced by the activity of the interferon-inducible cell surface protein BST-2/ CD317/HM1.24/"tetherin" [1-6]. The importance of overcoming this restriction for virus replication is reflected in the growing list of viral proteins that have been shown to possess anti-tetherin activities, with the primate lentiviruses in particular having evolved diverse approaches that include the HIV-1 Vpu, HIV-2 Env and certain SIV Nef and Env proteins [2,3,7-12].

Analyses of the interactions between tetherins from different primate species and the anti-tetherin proteins

\footnotetext{
* Correspondence: pcannon@usc.edu

Department of Molecular Microbiology and Immunology, Keck School of Medicine of the University of Southern California, Los Angeles, California,
} USA

(c) 2010 Yang et al; licensee BioMed Central Ltd. This is an Open Access article distributed under the terms of the Creative Commons Attribution License (http://creativecommons.org/licenses/by/2.0), which permits unrestricted use, distribution, and reproduction in any medium, provided the original work is properly cited. used by viruses that infect those hosts have revealed a high degree of specificity. For example, although all tetherins analyzed to date can block HIV-1 particle release as efficiently as human tetherin, non-human tetherins are usually insensitive to antagonism by the HIV-1 Vpu protein $[9,10,12-14]$. The determinants of the Vpu-tetherin interaction have been mapped to the transmembrane (TM) domain of tetherin $[9,13,15,16]$. Within $\mathrm{Vpu}$, the TM domain has long been known to be required for efficient virus release $[17,18]$ and is now known to play an important role in the Vpu-tetherin interaction [2,3], while the cytoplasmic tail of Vpu contains a $\beta$-TrCP binding domain comprising residues serine 52 and 56 and a positively charged hinge region at the start of the cytoplasmic domain which both contribute to its anti-tetherin activity $[14,19,20]$. In addition, specificity has been observed in the interaction between
C Biomed Central 
tetherins and SIV Nef proteins that depends on a short stretch of amino acids that is present in the cytoplasmic tail of primate tetherins such as chimpanzee, macaque, or African green monkey, but not in human tetherin $[9,10]$.

The primate lentiviruses have been classified into six major lineages on the basis of phylogenetic analyses (Table 1) [21,22]. Interestingly, only two lineages contain $\mathrm{Vpu}$ in their genome, the SIVcpz/HIV-1 lineage and certain members of the SIVsyk lineage that include the SIVgsn sublineage (SIVmus, SIVmon, and SIVgsn), as well as the SIVden isolate [23-28]. Vpu is a type I integral membrane protein that plays multiple roles in the HIV-1 life-cycle in addition to counteracting tetherin [29]. The close similarity between HIV-1 and SIVcpz led us to examine whether SIVcpz Vpu proteins could also counteract human tetherin, and if this could have been important in allowing HIV-1 to cross the species barrier and infect humans. Surprisingly none of the SIVcpz Vpu proteins that we tested had anti-tetherin activity, even against the species-matched chimpanzee tetherin. Instead, we found that an anti-tetherin activity in these viruses resides in the Nef protein. In contrast, the more distantly related SIVsyk viruses possessed an antitetherin activity in Vpu although, with a single exception, this was not active against human tetherin. Taken together, these findings suggest a high degree of flexibility in the evolution of anti-tetherin factors within the primate lentiviruses, with the diverse anti-tetherin strategies observed suggesting either convergent evolution or the re-acquisition of anti-tetherin activities in viral proteins as viruses adapted to new host species. It also leads us to speculate that having an anti-tetherin activity in Vpu in the SIVgsn ancestor that gave rise to the 3' half of the SIVcpz/HIV-1 genome may have been especially important for the evolution of the subgroup of viruses that could counteract human tetherin and infect humans.

\section{Results}

\section{Vpu from SIVcpz does not counteract human or chimpanzee tetherin}

SIVcpz viruses are closely related to HIV-1 and contain a Vpu open-reading frame (Figure 1A, Table 1). We tested whether SIVcpz Vpu proteins have anti-tetherin activity by examining their ability to increase the release of HIV-1 virus like particles (VLPs) from HeLa cells, which naturally express human tetherin $[2,3]$. We initially tested the Vpu protein from the GAB1 strain of SIVcpz, which is representative of viruses isolated from Pan troglodytes troglodytes that are more closely related to HIV-1, and also the Vpu protein from SIVcpz ANT, which is representative of the more distantly related viruses isolated from Pan troglodytes schweinfurthii
(Figure 1A) $[24,30]$. Both Vpu proteins were obtained as EGFP fusion proteins, whose functionality in CD4 down-regulation assays had previously been demonstrated [31]. Confocal microscopy revealed that the cellular distribution of GAB1 and ANT Vpu-EGFP (Figure 1B) resembled that which has been reported for HIV-1 $\mathrm{Vpu}[31,32]$. In addition, both GFP and YFP fusion proteins of HIV-1 Vpu have previously been shown to retain anti-tetherin activity $[2,19,33]$, and we confirmed the lack of effect of a C-terminal EGFP tag for HIV-1 $\mathrm{Vpu}$ by comparing the ability of Vpu and Vpu-EGFP to increase HIV-1 VLP release when expressed in HeLa cells (Figure 1C). Both proteins increased VLP release, and although the untagged $\mathrm{Vpu}$ construct had greater overall activity, this is likely a consequence of the higher levels of expression from this human codon-optimized construct. In contrast, neither of the two SIVcpz Vpu proteins had any effect on VLP release.

Since species specificities have been noted in the interaction between tetherin and viral anti-tetherin factors [9-11,13-15], we next examined whether the SIVcpz Vpu proteins had activity against chimpanzee tetherin. We expressed chimpanzee tetherin in human 293A cells which, similar to other derivatives of 293 cells, do not constitutively express human tetherin $[2,3]$. We found that chimpanzee tetherin was able to suppress HIV-1 VLP release just as efficiently as human tetherin. Furthermore, chimpanzee tetherin restriction was antagonized to a similar extent as human tetherin by both the HIV-1 Vpu and HIV-2 Env proteins (Figure 2A). However, neither of the SIVcpz Vpu proteins was able to increase HIV-1 VLP release in the presence of chimpanzee tetherin (Figure 2B). To rule out any requirements for other chimpanzee cellular factors, we also repeated these analyses by expressing chimpanzee tetherin in a chimpanzee B cell line. Although the HIV$1 \mathrm{Vpu}$ protein remained active against chimpanzee tetherin in this cell line, neither of the SIVcpz Vpus had any activity (Figure $2 \mathrm{C}$ ). Taken together, these results indicate that the $\mathrm{Vpu}$ protein from SIVcpz is not an antagonist of either human or chimpanzee tetherin.

\section{SIVcpz Env does not have anti-tetherin activity}

Other viral proteins in the primate lentiviruses that have been reported to have anti-tetherin activity include the Env proteins from HIV-2 and SIVtan $[7,8,12]$ and the Nef proteins from certain SIVs [9-11]. We examined the possibility that SIVcpz Env had anti-tetherin activity by generating HIV-1 VLPs in the presence of fragments of SIVcpz genomes comprising the Env, Vpu, and Rev proteins, in a configuration that we have previously shown can lead to expression of all three HIV-1 and HIV-2 proteins [7]. We performed these analyses on four additional SIVcpz isolates, spread throughout the lineage, 


\begin{tabular}{|c|c|c|c|}
\hline PLV lineages & Vpu? & Anti-tetherin factors previously reported & Proteins analyzed in this study \\
\hline \multirow[t]{7}{*}{ SIVcpz/HIV-1[23,24,44] } & + & HIV-1 Vpu[2,3] & HIV-1 NL4-3 Vpu \\
\hline & & & SIVcpz GAB1 Vpu \\
\hline & & & SIVcpz ANT Vpu \\
\hline & & & SIVcpz MT145 Env/Npu \\
\hline & & & SIVcpz TAN3 EnvNpu \\
\hline & & & SIVcpz MB897 Env/Npu, Nef \\
\hline & & & SIVcpz EK505 Env/Npu, Nef \\
\hline \multirow[t]{3}{*}{$\mathrm{SIVsm} / \mathrm{mac} / \mathrm{HIV}-2[45,46]$} & - & SIVsm Nef[9,10] & \\
\hline & & SIVmac239 Nef[9,10] & SIVmac239 Nef \\
\hline & & HIV-2 ROD10 Env[7-9,50] & HIV-2 ROD10 Env \\
\hline \multirow[t]{3}{*}{ SIVagm[47] } & - & SIVsab Nef[10] & \\
\hline & & SIVtan Nef[10,11] & \\
\hline & & SIVtan Env[11] & \\
\hline \multirow[t]{4}{*}{ SIVsyk[48] } & $++^{1}$ & SIVmus Vpu ${ }^{2}[11]$ & SIVmus 01CM2500 Vpu \\
\hline & & & SIVmon 99CMCML1 Vpu \\
\hline & & & SIVgsn 99CM71 Vpu \\
\hline & & & SIVden Vpu \\
\hline SIVI'hoest[49] & - & N/D & \\
\hline SIVcol[21] & - & N/D & \\
\hline
\end{tabular}

including MB897 and EK505 that are closely related to HIV-1 subtypes $M$ and N respectively, as well as MT145 and TAN3 which are more distantly related (Figure 1A) $[34,35]$. Due to the lack of specific antisera against these Env and Vpu proteins, we were only able to confirm the expression of three out of four Env proteins from these constructs (Figure 3A). None of the genomic fragments we tested exhibited anti-tetherin activity, either against endogenous human tetherin present in HeLa cells (Figure 3B), or in 293A cells transfected with chimpanzee tetherin (Figure $3 \mathrm{C}$ ). These data further confirm the lack of activity of the SIVcpz Vpu proteins and, additionally, reveal that SIVcpz Env proteins are not antitetherin factors.

\section{SIVcpz Nef counteracts chimpanzee but not human tetherin}

The absence of anti-tetherin activity in both SIVcpz Env and $\mathrm{Vpu}$ proteins led us to examine the Nef protein, since certain SIVs have previously been shown to have activity against tetherins from their host species that is a function of Nef (Table 1). We constructed Nef-EGFP proteins from SIVcpz MB897 and EK505, as well as from SIVmac239, since this has previously been reported to be active against macaque, but not human tetherin $[9,10]$. All three Nef-EGFP fusion proteins were expressed, although we noted that the SIVmac239 protein was present at lower steady-state levels (Figure 4A). We found that all three proteins were able to counteract chimpanzee tetherin when expressed in human 293A cells, with the least well expressed SIVmac239 protein having the greatest activity. This indicates that for the SIVcpz viruses, Nef fulfills the role of anti-tetherin factor (Figure 4B). In contrast, none of the Nef proteins had activity against human tetherin expressed in the same cells (Figure 4C). This finding agrees with observations recently reported by Sauter et al., who demonstrated that other SIVcpz isolates, including SIVcpzGAB1 and ANT, also contain a functional antitetherin activity in their Nef proteins [36].

The specificity of SIVmac Nef for macaque, but not human tetherin, has previously been reported to be conferred by the presence of 5 additional residues in the cytoplasmic tail of macaque tetherin, G/D-DIWK $[9,10]$. We asked whether this same sequence was responsible for the specificity observed in the SIVcpz Nef proteins by creating a chimeric human tetherin containing an insert of the equivalent chimpanzee residues, $\mathrm{H}(+5)$ tetherin (Figure 5A). We confirmed that $\mathrm{H}(+5)$ was expressed (Figure 5B) and fully functional in suppressing 
A

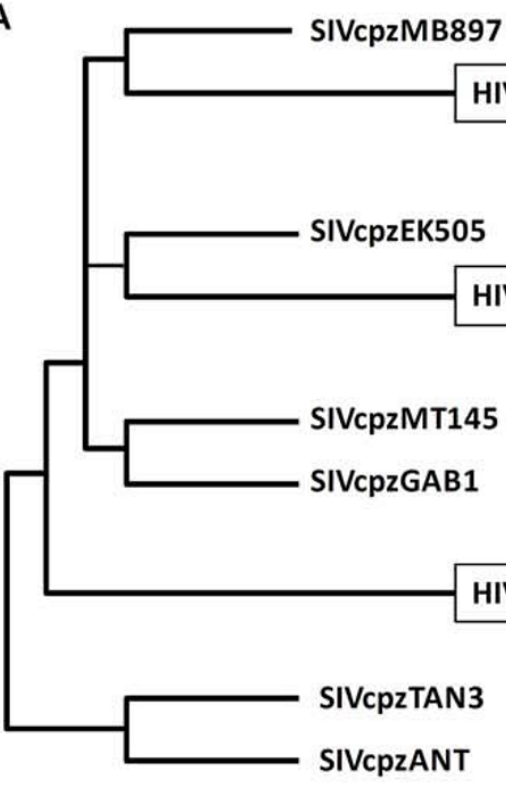

HIV-10

C

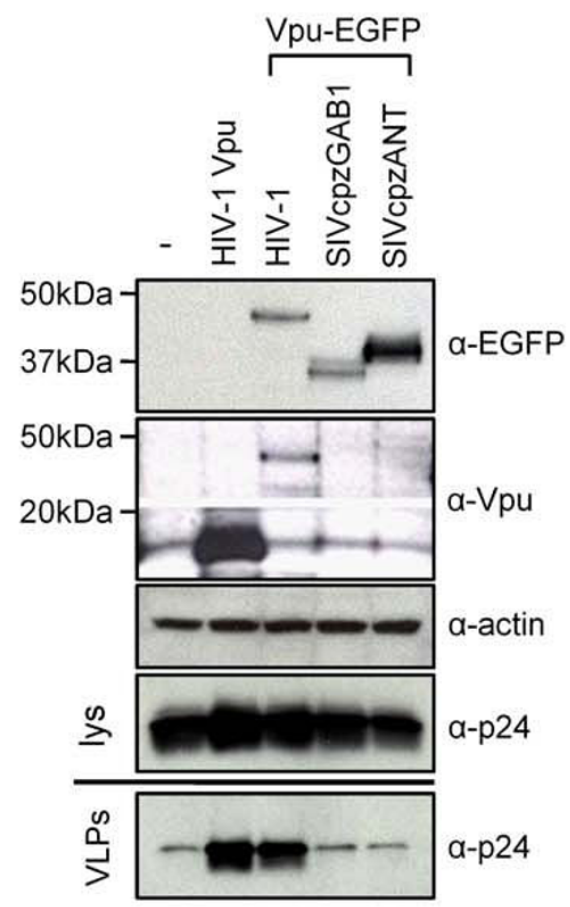

B

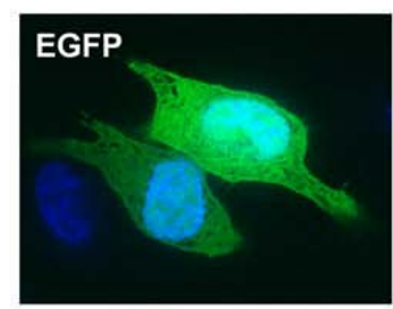

P.t.t.
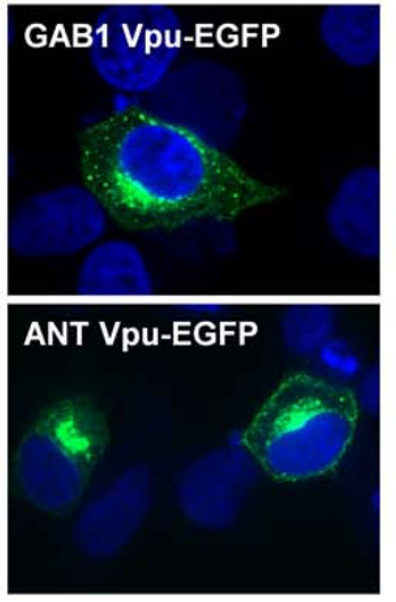

P.t.s.
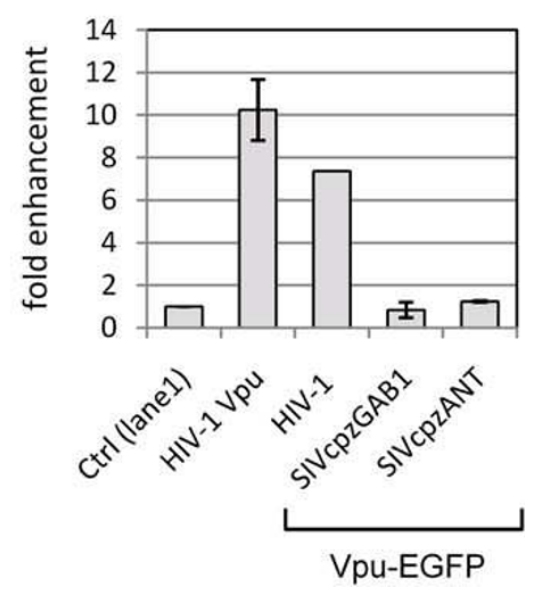

Figure 1 HIV-1 but not SIVcpz Vpu overcomes human tetherin restriction. (A) SIVcpz/HIV-1 lineage of the primate lentiviruses, showing three major HIV-1 groups (M, N and O) and the SIVcpz isolates used in this study. SIVcpz TAN3 and ANT were isolated from Pan troglodytes schweinfurthii (P.t.s.) and are less closely related to HIV-1 than SIVcpz strains isolated from Pan troglodytes troglodytes (P.t.t.). Figure adapted from Wain et al. (2007) [35]. (B) Confocal analysis of distribution of GAB1 and ANT Vpu-EGFP fusion proteins and EGFP control, in transiently transfected HeLa cells. (C) HeLa cells (express tetherin) were transfected with pHIV-1-pack (expresses HIV-1 Gag-Pol, Rev), together with either a control CMV expression vector (-), or expression plasmids for human codon-optimized Vpu from HIV-1 (HIV-1 Vpu), or non-codon-optimized EGFP tagged Vpu proteins from HIV-1, SIVcpz GAB1 or SIVcpz ANT. Cell lysates (lys) were probed with indicated antibodies. The Vpu-EGFP proteins from HIV-1, SIV Cpz GAB1 and SIVcpz ANT have predicted molecular weights of 47, 33 and 42 kDa, respectively. Intracellular Gag proteins in cell lysates and virus-like particles released into supernatant (VLP) were detected using anti-p24 antibody. Mean-fold enhancement of HIV-1 VLP release in presence of Vpu is shown relative to baseline (control) levels in absence of Vpu for three independent experiments, except for the HIV-1 Vpu-EGFP sample $(n=1)$. 


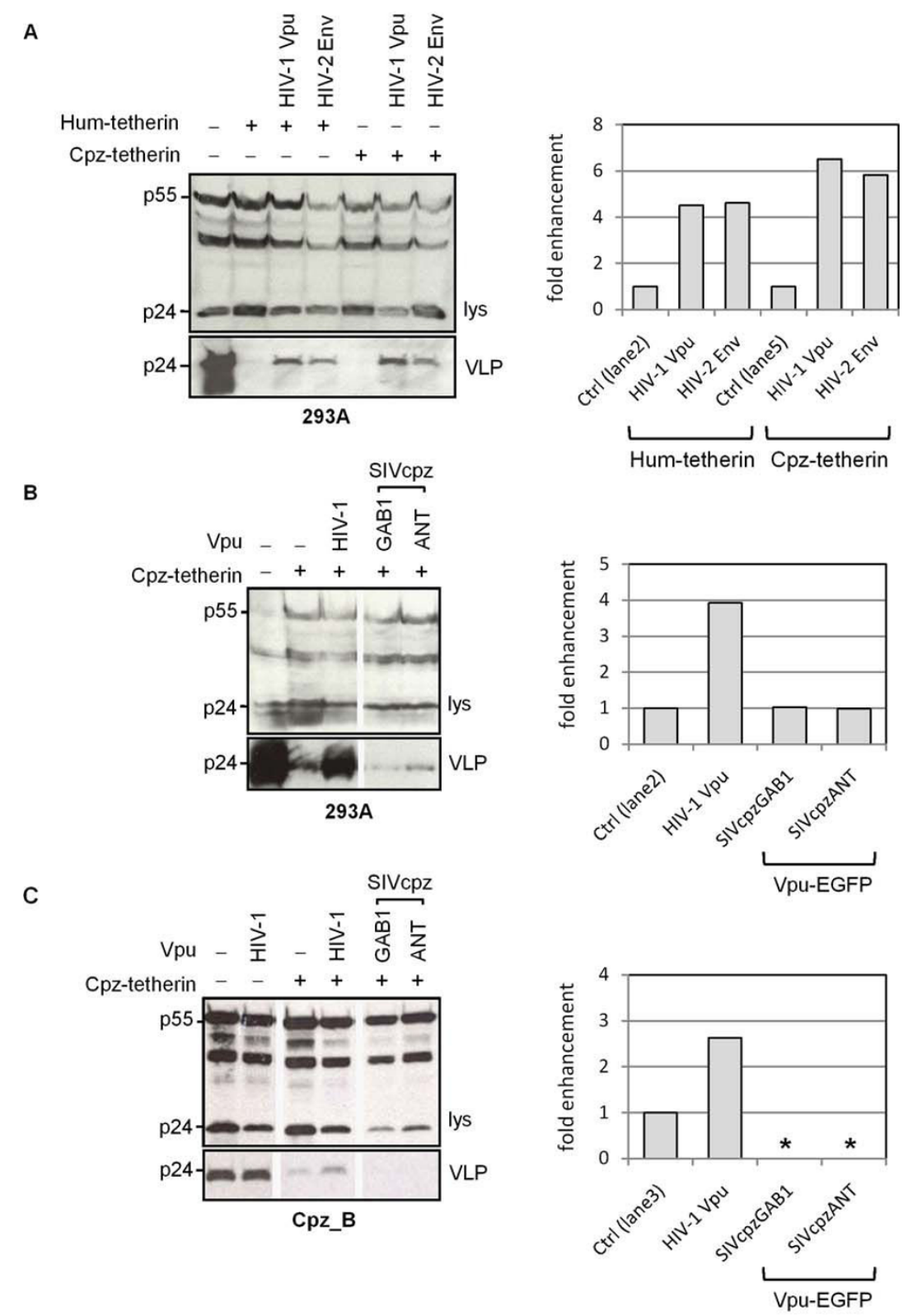

Figure 2 Activity of HIV-1 and SIVcpz Vpu against chimpanzee tetherin. Anti-tetherin activities of indicated viral proteins were examined by measurement of HIV-1 VLP release, detected by Western blotting of cell lysate and VLP fractions with anti-p24 antibody (left panels) or as meanfold enhancement of VLP release relative to baseline (control) levels in absence of Vpu or Env proteins (right panels): (A) HIV-1 Vpu and HIV-2 Env activity against human (Hum) and chimpanzee (Cpz) tetherin expressed in 293A cells, (B) Activity of HIV-1 Vpu and SIVcpz GAB1 and SIVcpz ANT Vpu-EGFP proteins against Cpz-tetherin expressed in 293A cells, and (C) Activity of HIV-1 Vpu and SIVcpz GAB1 and SIVcpz ANT Vpu-EGFP proteins against Cpz-tetherin expressed in chimpanzee (Cpz_B) cells. * indicates p24 signal was too low to quantify.

virus release when transfected into 293A cells (Figure $5 \mathrm{C})$. The presence of these 5 additional residues was sufficient to make human tetherin a target for both SIVmac239 Nef and the SIVcpz Nef proteins (Figure 5C), indicating that the observed species specificity of the interaction between the SIVcpz Nef proteins and tetherin involves the same target sequence as SIVmac Nef.

\section{Anti-tetherin activity of SIVsyk Vpu}

Certain members of the SIVsyk lineage express Vpu, specifically those from the SIVmus/mon/gsn sub-lineage [25-27], and also the SIVden isolate [28] (Figure 6A). We analyzed the anti-tetherin activity of Vpu proteins from representatives of each of these four groups within the SIVsyk lineage. Each SIV Vpu was constructed as an EGFP fusion protein, and the expression of each protein 


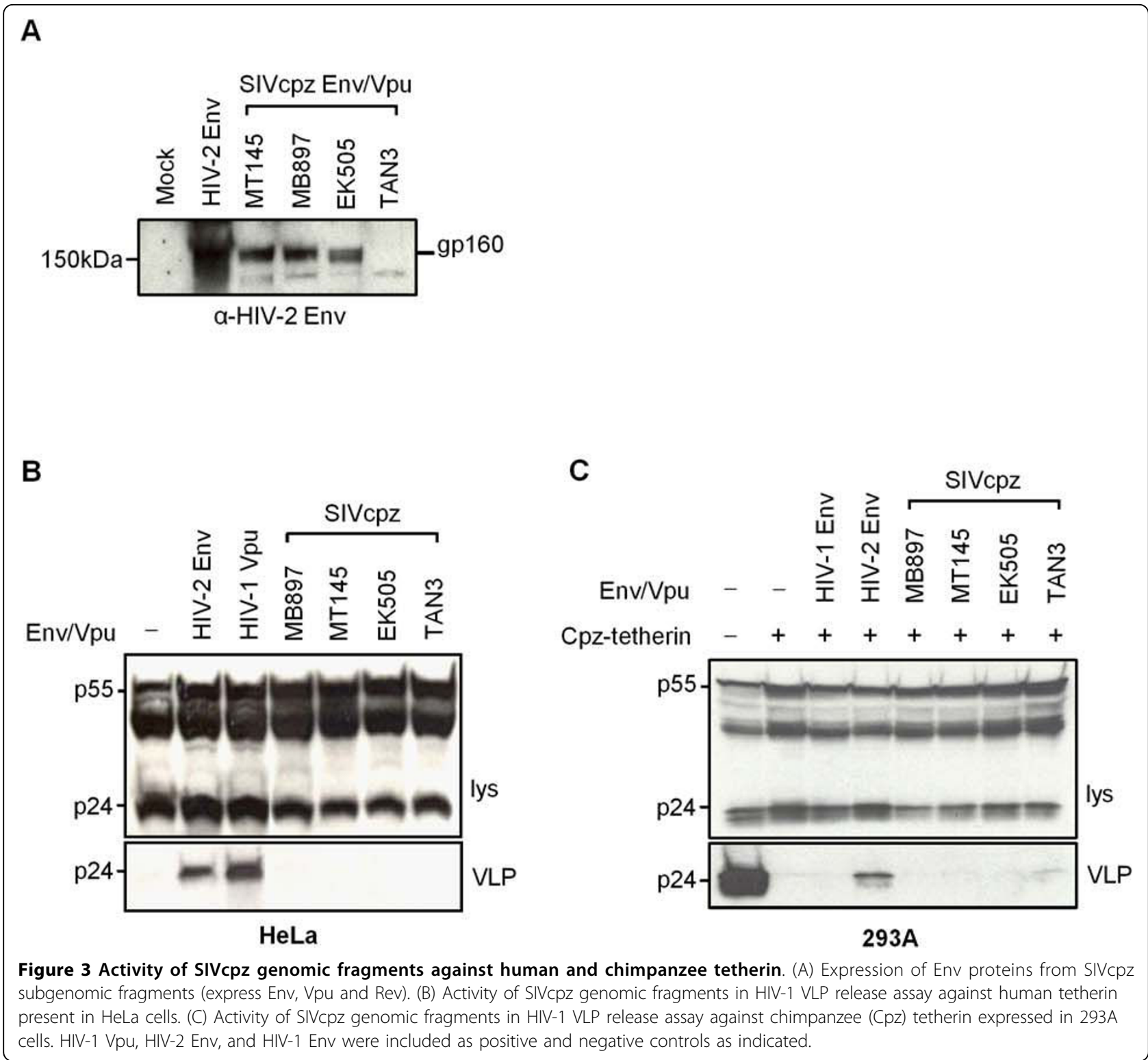

was analyzed by Western blotting, where we observed some differences in steady-state levels (Figure 6B). We analyzed their activity against both human and macaque tetherin, since macaques are more closely related to the Old World primate hosts of these viruses. Macaque tetherin has previously been shown to be resistant to HIV-1 Vpu, but counteracted by SIVmac239 Nef $[9,10]$ and HIV-2 Env [9], and we confirmed these specificities (data not shown). Anti-tetherin activity was assessed in both HeLa cells, (Figure 6C), and in macaque LLCMK2 cells transiently transfected with macaque tetherin (Figure 6D). We found that all four SIV Vpu proteins were able to counteract the inhibition of VLP release caused by macaque tetherin, which is in agreement with a recent report [36]. We cannot rule out that the greater activity of the SIVmus and SIVgsn Vpu-EGFP proteins could arise from their higher expression levels. In contrast, only the Vpu from SIVgsn (strain 99CM71) showed activity against human tetherin. Our finding of activity against human tetherin in this SIVgsn isolate is the first report of such an activity in a naturally occurring SIV Vpu protein.

To address whether the lack of activity of the Vpu proteins from SIVmus/mon/den in HeLa cells was caused by incompatibility between their Vpu proteins and human tetherin or, instead, reflected some other differences between human and macaque cells, we repeated these analyses expressing human tetherin in LLCMK2 cells. Similar to our findings in HeLa cells we observed that only SIVgsn Vpu had activity against 


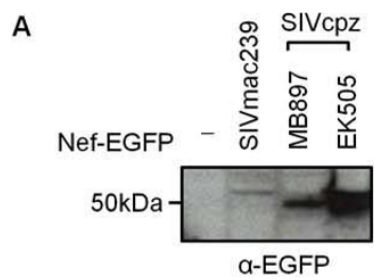

B
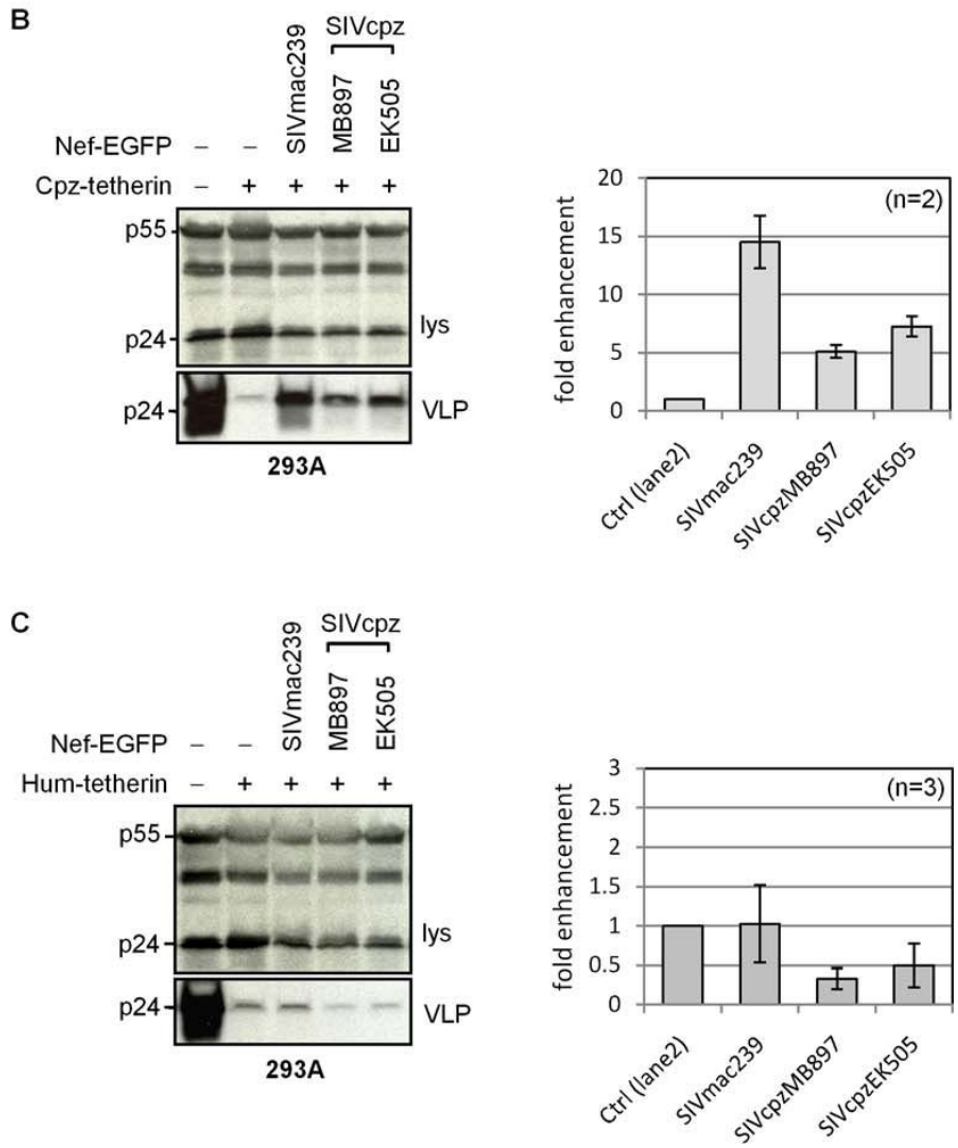

Figure 4 Anti-tetherin activity of SIVcpz Nef. (A) Expression of indicated SIV Nef-EGFP proteins, detected with anti-GFP antibody. Activities of Nef-EGFP proteins against (B) chimpanzee, or (C) human tetherin expressed in 293A cells. Mean fold-enhancement of HIV-1 VLP release in presence of Nef-EGFP is shown relative to baseline (control) levels for $n=2$ or 3 independent experiments.

human tetherin in this cell background (Figure 6E). SIVgsn 99CM71 Vpu is therefore an example of a naturally existing protein from a non-human primate lentivirus that has activity against human tetherin.

\section{Specificity of interaction between SIVsyk Vpu proteins and tetherin}

Since the SIVsyk Vpu proteins displayed species specificities in their interactions with tetherin, we asked whether this mapped to the same region of tetherin responsible for the specificity of interaction with SIVmac and SIVcpz Nef proteins, by examining their activity against the chimeric protein $\mathrm{H}(+5)$-tetherin (Figure $5 \mathrm{~A}$ ). In contrast to the situation with the SIVcpz Nef proteins, where the addition of these amino acids to the cytoplasmic tail of human tetherin conferred sensitivity (Figure 5C), we found that the $\mathrm{H}(+5)$-tetherin remained resistant to all of the SIVsyk Vpu proteins except SIVgsn (Figure 7A), which was unsurprising, given its activity against both human and macaque proteins (Figure 6).

The species-specificity of the interaction between the HIV1 Vpu protein and tetherin has been mapped to the TM domain of both proteins $[2,3,9,13,15,16]$. We therefore replaced both the $\mathrm{N}$-terminal cytoplasmic tail and TM regions of human tetherin with the corresponding macaque sequences to create $\mathrm{MH}$ tetherin (Figure 7B) 


\begin{tabular}{|c|c|c|c|}
\hline A & $\mathrm{N}$-terminus & TM & Extracellular \\
\hline Human & MASTSYDYCRVPM-----EDGDKRCK & LLLGIGILVLLI IVILGVPLIIFT & IKANSEACRD... \\
\hline $\mathrm{Cpz}$ & MASTLYDYCRVPMDDIWKKDGDKRCK & LLLGIGILMLLI IVILGVPLIIFT & IKANSEACRD ... \\
\hline$H(+5)$ & MASTSYDYCRVPMDDIWKKDGDKRCK & LLLGIGILVLLI IVILGVPLIIFT & IKANSEACRD... \\
\hline
\end{tabular}

B

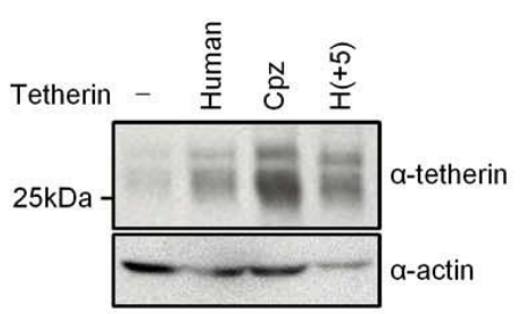

C

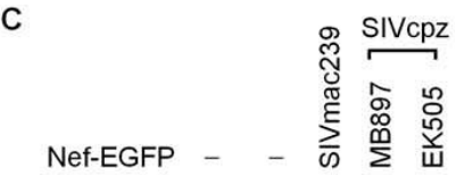

$\mathrm{H}(+5)$-tetherin -++++
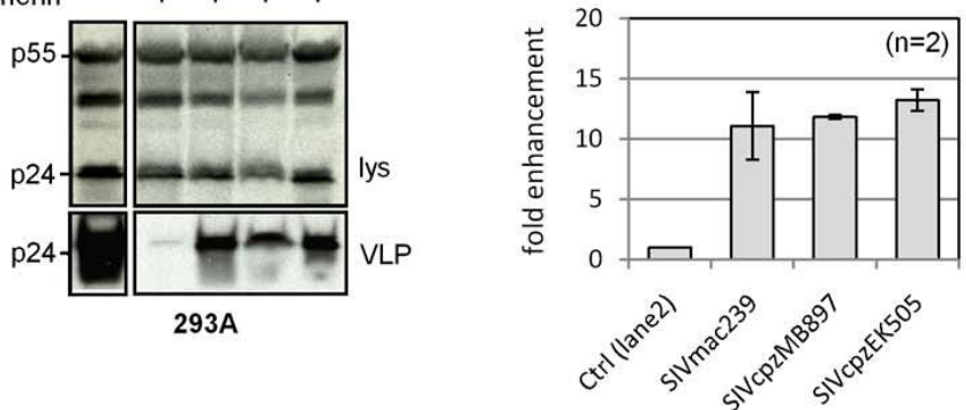

Figure 5 Specificity of interaction between SIVcpz and SIVmac Nef proteins and tetherin. (A) Partial sequence alignment of human, chimpanzee $(\mathrm{Cpz})$ and $\mathrm{H}(+5)$-tetherin proteins, showing the N-terminal cytoplasmic tail, the transmembrane (TM) domain and the start of the extracellular domain. $\mathrm{H}(+5)$-tetherin contains an insertion (DDIWKK) from Cpz-tetherin in place of human tetherin residue E-14. (B) Western blot of expression of indicated tetherin constructs, from lysates of transfected 293A cells. (C) Anti-tetherin activities of SIVmac239 and SIVcpz Nef-EGFP proteins against $\mathrm{H}(+5)$-tetherin expressed in 293A cells. Mean fold-enhancement of HIV-1 VLP release in presence of Nef-EGFP proteins is shown relative to baseline (control) levels for $n=2$ independent experiments.

and confirmed protein expression (Figure 7C). We observed that all of the SIVsyk Vpu proteins, but not HIV-1 Vpu, had activity against this chimeric protein (Figure 7D). This suggests that, similar to the situation with HIV-1 Vpu, the specificity of the interaction between SIVsyk Vpu proteins and tetherin also maps to the TM domain.

\section{SIVgsn Vpu removes human tetherin from the surface of HeLa cells}

We next asked whether the SIVgsn Vpu was able to remove tetherin from the cell surface, as we and others have previously observed for HIV-1 Vpu and human tetherin $[3,14,16,20,33,37]$. As controls we also included Vpu proteins from the SIVmus and SIVmon strains that were not active against human tetherin. Confocal analysis of cell surface tetherin on HeLa cells transfected with Vpu-EGFP fusion proteins demonstrated the removal of tetherin by only the HIV-1 and SIVgsn Vpu proteins (Figure 8A), and FACS analysis confirmed these observations (Figure 8B). Therefore, the ability to counteract human tetherin correlates with its removal from the cell surface for both HIV-1 and SIVgsn Vpu. 
A

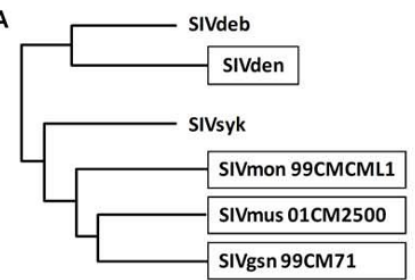

C

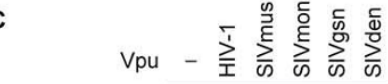

HeLa

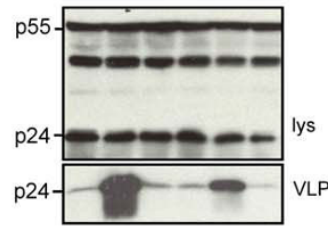

D

离 $\mathrm{vpu}$

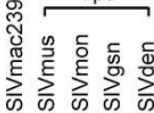

क के के

Mac-tetherin -++++++

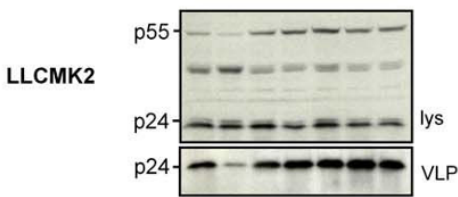

E

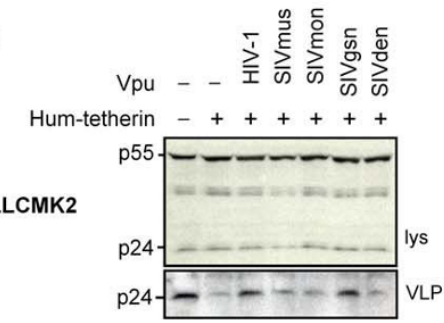

B

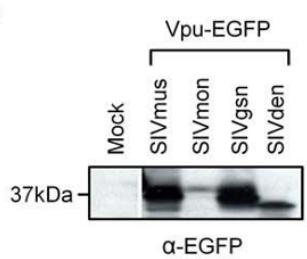

sublineage

$\alpha$-EGFP
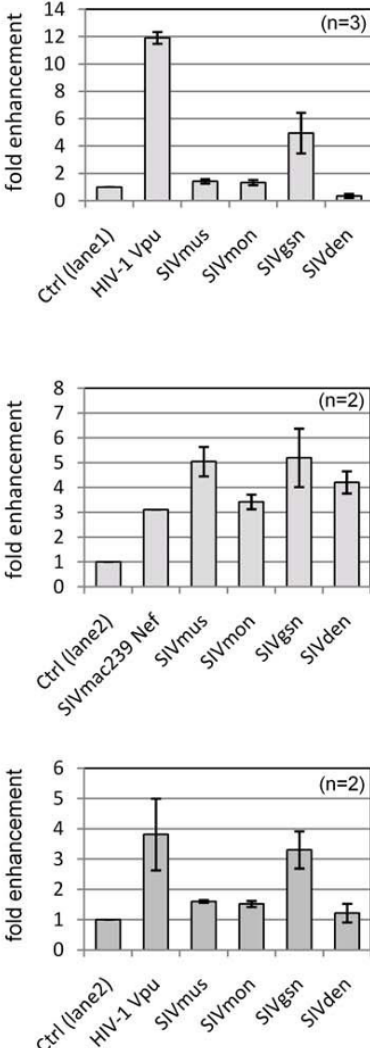

Figure 6 Anti-tetherin activity of SIVsyk lineage Vpu proteins. (A) SIVsyk lineage of the primate lentiviruses, showing viruses that express Vpu (boxed). SIVmon/mus/gsn viruses form the SIVgsn sublineage, while SIVden is less closely related. Figure adapted from Dazza et al. (2005) [28]. (B) Expression of SIVmus/mon/gsn and SIVden Vpu-EGFP proteins detected with anti-GFP antibody. Anti-tetherin activities of indicated VpuEGFP proteins were measured by HIV-1 VLP release assays, against (C) human tetherin present in HeLa cells, (D) Mac-tetherin expressed in macaque (LLCMK2) cells, and (E) Hum-tetherin expressed in LLCMK2 cells. HIV-1 Vpu and SIVmac239 Nef-EGFP proteins were included as controls. Mean fold-enhancement of HIV-1 VLP release in presence of anti-tetherin proteins is shown relative to baseline (control) levels, for $n=$ 2 independent experiments.

\section{Discussion}

The primate lentiviruses exhibit a high degree of flexibility in their ability to counteract the BST-2/tetherin restriction of virus release. To date, three different proteins (Vpu, Env and Nef) have been shown to act as anti-tetherin factors in different primate lentiviruses, which highlights the importance of an anti-tetherin activity for their life-cycle. The HIV-1 Vpu protein is the prototypical anti-tetherin factor, and the observation that mature virions remain attached at the cell surface if tetherin is not counteracted was first observed for Vpu- minus HIV-1 [38]. The paradox of such an activity being associated with a protein that is not present in the genome of the other lineage of lentiviruses that infect humans, HIV-2, was resolved when a virus releaseenhancing activity was mapped to the HIV-2 Env protein $[8,39]$. More recently, several different strains of SIV have been shown to carry anti-tetherin activities in either Nef or Env proteins [9-12,36].

The Vpu open-reading frame is not unique to HIV-1 but is also present in all other members of the SIVcpz/ HIV-1 lineage. In addition, certain members of the 
SIVsyk viruses (the SIVgsn sublineage and SIVden) code for $\mathrm{Vpu}$. We therefore asked the question, if $\mathrm{Vpu}$ is present, does it always exhibit anti-tetherin activity? Interestingly, despite the close relationship between HIV-1 and the SIVcpz viruses, we found that the Vpu proteins from SIVcpz do not have activity against either chimpanzee, macaque or human tetherin. Instead, we determined that these viruses have evolved to target chimpanzee tetherin using their Nef protein, a finding that has also recently been reported [36]. In this way, these viruses are more similar to other SIV strains, specifically SIVsm/mac and SIVagm, that also possess anti-tetherin activities in Nef [9-11]. Primate tetherins, including chimpanzee, macaque and African green monkey, differ from the human protein by having an additional sequence of 5 amino acids (G/DDIWK) in their cytoplasmic tail, which has previously been shown to be necessary for SIVmac Nef to counteract primate tetherins $[9,10]$. Similarly, we have now confirmed that this motif is also essential for the recognition of primate tetherins by SIVcpz Nef proteins. It therefore seems likely that the use of $\mathrm{Vpu}$ by HIV-1 and Env by HIV-2 was necessitated, in part, because Nef cannot easily target human tetherin in the absence of this motif.

The fact that SIVcpz strains maintain the Vpu ORF, despite its lack of activity against chimpanzee tetherin, probably reflects the fact that $\mathrm{Vpu}$ is a multi-functional protein [29]. Indeed, down-regulation of CD4 by SIVcpz $\mathrm{Vpu}$ proteins has been confirmed by others $[31,36,40]$. Thus, Vpu's ability to target CD4 is well conserved in the SIVcpz/HIV-1 lineage, while the anti-tetherin activity may be a more recently acquired, or re-acquired, activity in the viruses that infect humans.

In contrast to the situation with SIVcpz, we found that Vpu proteins from the SIVsyk viruses are capable of antagonizing tetherin restriction. Within the SIVsyk lineage, there is a close phylogenetic relationship between the SIVmus/mon/gsn viruses, which form a separate sublineage termed SIVgsn $[24,26,28]$. A less closely related virus, SIVden, also expresses Vpu, although the protein is 10 amino acids shorter than $\mathrm{Vpu}$ from the SIVmus/mon/gsn viruses [28]. Representative $\mathrm{Vpu}$ proteins from all four groups of viruses were tested and found to be capable of overcoming the restriction mediated by macaque tetherin. Consistent with our observations, Lim et al. have also found that SIVmus $\mathrm{Vpu}$ antagonizes African green monkey and mustached monkey tetherins [11]. In addition, while this manuscript was in preparation, Sauter et al. also reported that SIVmus/mon/gsn Vpu proteins have anti-tetherin activities against host species-matched tetherins, with the specificity determined by the TM domain of tetherin [36].
An interesting finding from our studies of the SIVsyk lineage was that the Vpu protein from SIVgsn 99CM71 was also capable of antagonizing human tetherin. This activity was confirmed by both confocal studies and FACS analyses, where we observed that similar to the HIV-1 Vpu, SIVgsn Vpu removed tetherin from the surface of human cells. The presence of an anti-tetherin activity that is active against the human form of the protein in this SIVgsn virus supports the hypothesis that the SIVcpz/HIV-1 lineage arose by recombination, with the $5^{\prime}$-half of the genome originating from SIVrcm and the 3'-half, that includes $\mathrm{Vpu}$, deriving from the SIVgsn sublineage $[30,41]$. Consequently, a recombinant ancestor of HIV-1 could have contained a Vpu protein with some capability of targeting human tetherin. At the same time, the lack of the G/D-DIWK motif in the cytoplasmic tail of human tetherin would have restricted the adoption of Nef for this activity, as occurred in the SIVcpz viruses. Since the interactions between Vpu and tetherin are highly specific, $[9,10,13-15]$, it is likely that the acquisition of the ability to target human tetherin in HIV-1 Vpu would have resulted in the loss of any ability to target Old World primate tetherins, leading to the present day restriction of HIV-1 Vpu's activity for human tetherin.

\section{Conclusions}

The ability to counteract tetherin restriction appears to be an essential activity of primate lentiviruses. At least three different proteins have evolved in different virus backbones and host environments to target this host cell restriction. Although several diverse SIVs use Nef to target tetherin, including the SIVcpz viruses that are closely related to HIV-1, the lack of a 5 residue sequence in the cytoplasmic tail of human tetherin makes it a difficult target for Nef. Possibly, this led to the adoption of alternate anti-tetherin approaches in the human immunodeficiency viruses based on Vpu (HIV-1) and Env (HIV-2). For HIV-1, we speculate that the presence of anti-tetherin activity in the Vpu protein from the SIVgsn ancestor that contributed the 3'-half of the SIVcpz/HIV-1 genome allowed HIV-1 to evolve such an activity in $\mathrm{Vpu}$, and contributed to the ability of this virus to successfully infect humans.

\section{Methods \\ Cell lines}

HeLa and LLCMK2 (macaque) cells were obtained from the American Type Culture Collection; 293A cells were obtained from Qbiogene/MP Biomedicals (Irvine, CA). All cells were maintained in DMEM (Mediatech, Herndon, VA) supplemented with $10 \%$ fetal bovine serum (FBS) (Mediatech) and $2 \mathrm{mM}$ glutamine (Gemini BioProducts, West Sacramento, CA). The Cpz-B 
A

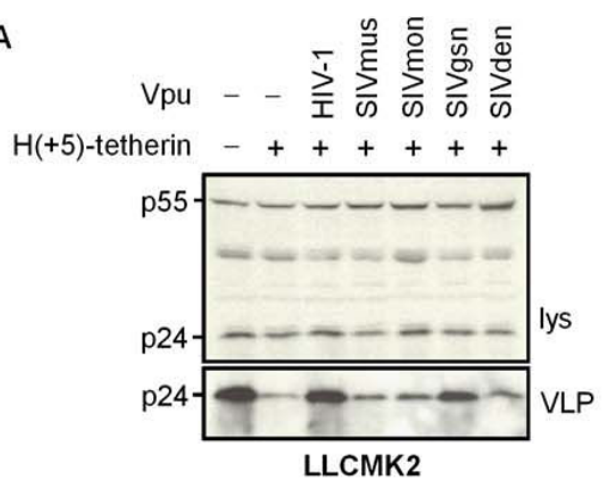

B

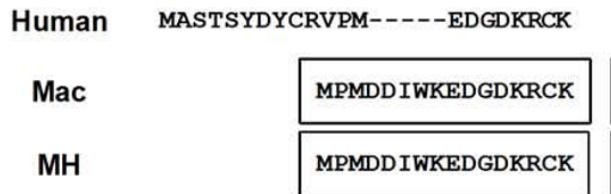

C

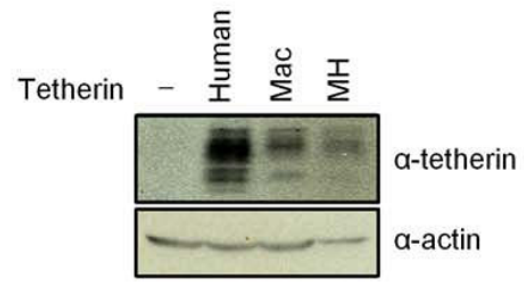

D

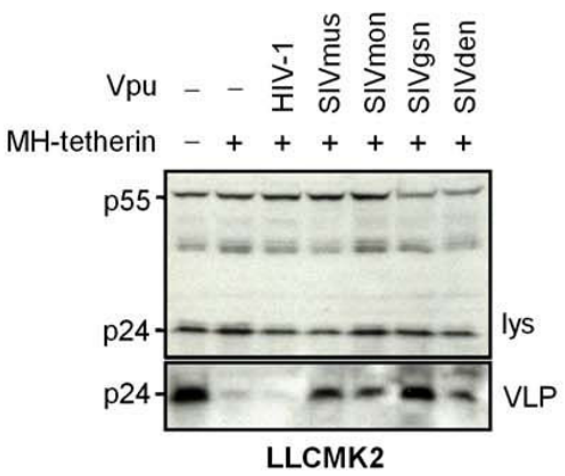

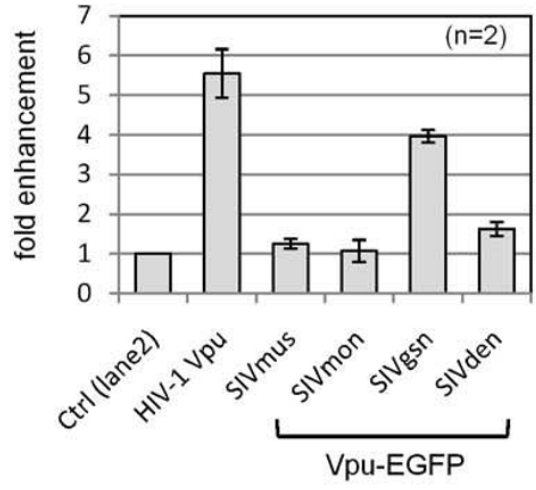

TM

Extracellular

LLLGIGILVLLI IVILGVPLIIFT

IKANSEACRD...

LVIGI--LGLLVIVLLGVLLIFFI

IKANSEACQD...

LVIGI--LGLLVIVLLGVLLIFFI

IKANSEACRD...

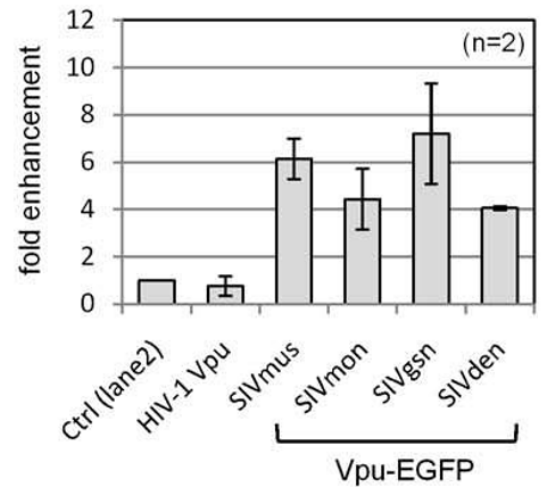

Figure 7 Specificity of interaction of SIVsyk Vpu proteins and tetherin. (A) Anti-tetherin activities of HIV-1 Vpu and indicated SIVsyk VpuEGFP proteins against H(+5)-tetherin expressed in LLCMK2 cells, measured by HIV-1 VLP release assay. Mean fold-enhancement of HIV-1 VLP release in the presence of anti-tetherin proteins is shown relative to baseline (control) levels in their absence, for $n=2$ independent experiments. (B) Partial sequence alignment of human, macaque (Mac) and $\mathrm{MH}$-tetherin showing the $\mathrm{N}$-terminal cytoplasmic tail, the transmembrane (TM) domain and the start of the extracellular domain. Mac-tetherin starts at M-11 of the full-length Macaca mulatta tetherin [9]. MH-tetherin has N-terminal cytoplasmic tail and TM domains of Mac-tetherin with a human tetherin extracellular domain. (C) Western blot of expression of indicated tetherin constructs, from lysates of transfected 293A cells. (D) Anti-tetherin activities of HIV-1 Vpu and indicated SIVsyk Vpu-EGFP proteins against MH-tetherin expressed in LLCMK2 cells, measured by HIV-1 VLP release assay. Mean fold enhancement of VLP release in presence of Vpu proteins is shown relative to baseline (control) levels in absence of Vpu, for $n=2$ independent experiments. 


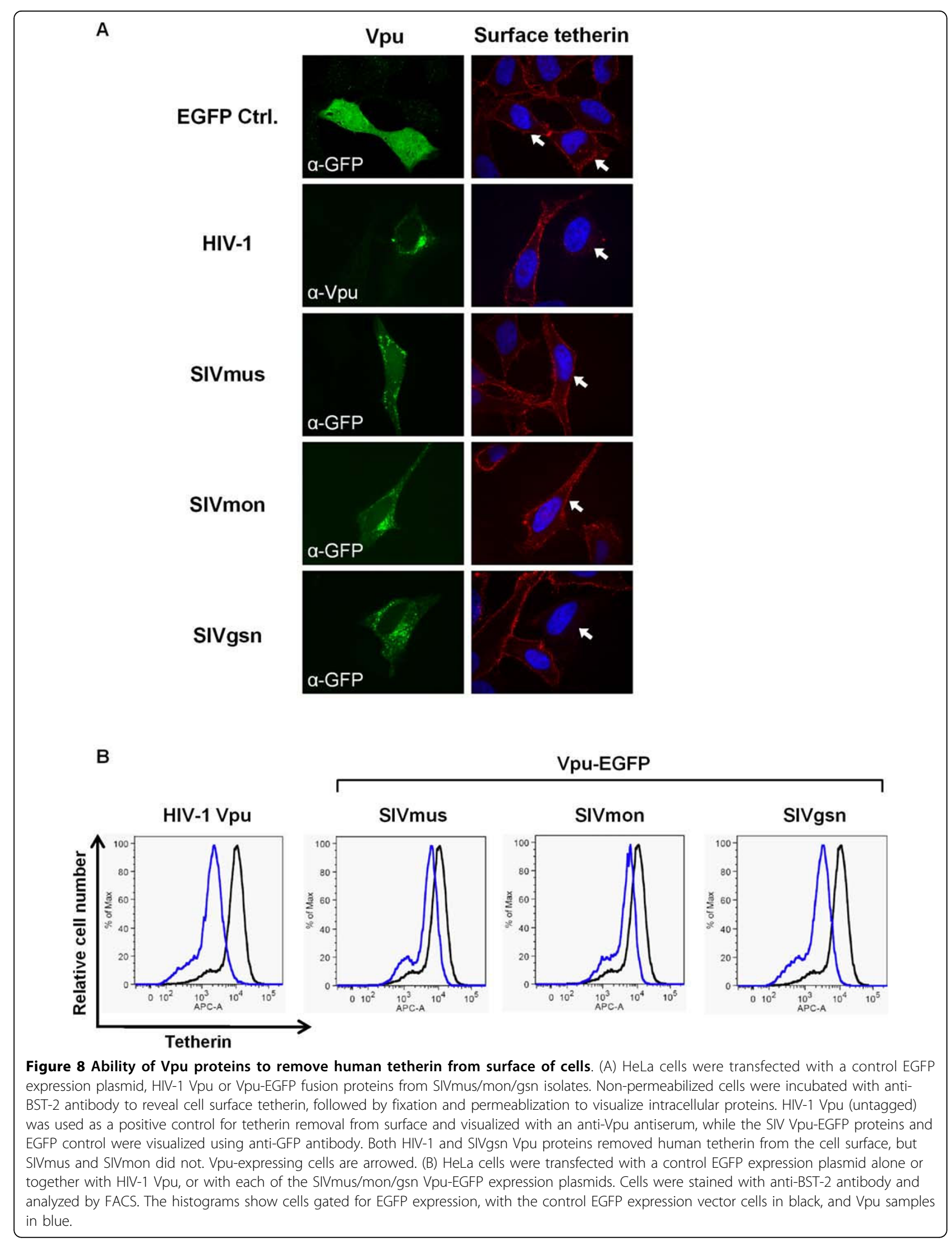


(chimpanzee) cell line was kindly provided by Jae Jung (University of Southern California) and cultured in RPMI media (Invitrogen, Carlsbad, CA) with 10\% FBS and $2 \mathrm{mM}$ glutamine.

\section{Plasmids}

Plasmid pHIV-1-pack expresses HIV-1 Gag-Pol and Rev [7]. Plasmid pcDNA-Vphu (HIV-1 Vpu) encodes a human codon-optimized form of Vpu from HIV-1 isolate NL4-3, kindly provided by Klaus Strebel (NIH) [42]. Expression plasmids for the Env proteins from HIV-2 (isolate ROD10, HIV-2 Env) and HIV-1 isolate BH10 (HIV-1 Env) have been previously described [7]. Expression plasmids containing Vpu-EGFP fusion proteins from SIVcpz isolates GAB1 and ANT were kindly provided by Edward Stephens (University of Kansas) [31]. Vpu-EGFP fusion proteins from SIVmus 01CM2500 (GenBank: ABO61057), SIVmon 99CMCML1 (GenBank: AAR02384), SIVgsn 99CM71 (GenBank: AAM90227) and SIVden (GenBank: CAE46404) were synthesized as human codon-optimized open-reading frames (Bio Basic Inc. Ontario, Canada) and fused to EGFP by cloning into vector pAcEGFP-N1 (Clontech, Mountain View CA). Fragments of SIVcpz genomes spanning the Vpu-Env-Rev open reading frames (SIVcpz Env/Vpu) were PCR amplified and cloned into the immediate-early CMV promoter expression vector, pSA91. The SIVcpz isolates used were MT145 (GenBank: DQ373066; co-ordinates 5495 to 8436), MB897 (GenBank: EF535994; coordinates 5519 to 8369), EK505 (GenBank: DQ373065; co-ordinates 5522 to 8346), and TAN3 (GenBank: EF394358; co-ordinates 5603 to 8608), generously provided by Beatrice Hahn (University of Alabama). The Nef proteins from SIVmac239 (GenBank: AAU14056), SIVcpz MB897 (GenBank: ABU53024), and SIVcpz EK505 (GenBank: ABD19500) were cloned as NefEGFP fusion proteins in vector pAcEGFP-N1. A human BST-2/tetherin (Hum-tetherin) expression plasmid (pCMV6-XL5-Bst2) was obtained from Origene (Rockville, MD), and the corresponding tetherin from Pan troglodytes (Cpz-tetherin, GenBank: XP_512491), was generated by site-directed mutagenesis of the human protein. Macaca mulatta tetherin (Mactetherin, GenBank: ACV96781) starting at methionine11 , which corresponds to $\mathrm{rBST} 2 \Delta 10$, and retains full tetherin activity and sensitivity to SIVmac239 Nef [9], was cloned by RT-PCR from total RNA isolated from LLCMK2 cells and inserted into vector pCMV6-XL5 (Origene). The chimeric tetherin $\mathrm{H}(+5)$-tetherin is human tetherin with the sequence DDIWKK replacing amino acid E-14, while $\mathrm{MH}$-tetherin comprises amino acids 11-38 of $M$. mulatta tetherin spliced to amino acid isoleucine- 46 of human tetherin.

\section{Production and analysis of HIV-1 VLPs}

HIV-1 VLPs were generated from HeLa, 293A, or LLCMK2 cells by transient transfection of $80-90 \%$ confluent cells in $10 \mathrm{~cm}$ plates with $8 \mu \mathrm{g}$ of pHIV-1-pack, together with any additional specified plasmids, using Lipofectamine 2000 (Invitrogen, Carlsbad, CA). The following amounts of plasmid DNA were used: $2 \mu \mathrm{g}$ of HIV-1 Vpu, HIV-1 BH10 Env, HIV-2 ROD10 Env, SIVcpz Env/Vpu, SIVmus/mon/gsn/den Vpu-EGFP; 5 $\mu \mathrm{g}$ of HIV-1, SIVcpzGAB1, or SIVcpzANT Vpu-EGFP; $0.5 \mu \mathrm{g}$ of SIVmac239 and SIVcpz Nef-EGFP. Cpz_B chimpanzee cells were nucleofected using an Amaxa Nucleofector series X (Amaxa, South San Francisco, CA) using the pre-set program U-08. Cell lysates and viral particles were collected 24 or 48 hours post-transfection and analyzed by Western blotting, as previously described [43]. HIV-1 p24-reacting proteins were detected using rabbit HIV-1 SF2 $_{\text {p } 24}$ Antiserum (AIDS Research and Reference Reagent Program, ARRRP) at a 1:3,000 dilution, followed by horseradish peroxidase (HRP)-conjugated goat anti-rabbit IgG $(1: 10,000)$ (Santa Cruz Biotechnology Inc., Santa Cruz, CA). Specific proteins were visualized using the enhanced chemiluminescence (ECL) detection system (Amersham International, Arlington Heights, IL). Exposed and developed films were scanned and quantified using the public domain NIH Image software. The intensity of the CA-reacting bands on the Western blots was measured and the ratio of the signal in virions:lysates obtained. The foldenhancement of virus budding was calculated by normalizing all values to the pHIV-1-pack only control.

\section{Western blotting}

Expression of all Vpu-EGFP and Nef-EGFP fusion proteins was detected by Western blotting of cell lysates using rabbit anti-GFP at a 1:1000 dilution (Santa Cruz Biotechnology Inc.). Env expression from the SIVcpz subgenomic constructs was detected using rabbit antiHIV-2 Env antiserum at a 1:1000 dilution (ARRRP). Expression of the various tetherin constructs was detected using rabbit anti-BST-2 at a 1:30,000 dilution (ARRRP, from Klaus Strebel). Actin was included as a loading control, and detected using a mouse anti-actin antibody (Sigma Aldrich, St. Louis, MO) at a dilution of 1:15,000. Secondary antibodies used were HRP-conjugated anti-rabbit IgG at a 1:10,000 (Santa Cruz Biotechnology Inc) or HRP-conjugated anti-mouse IgG at a 1: 10,000 (Sigma Aldrich).

\section{Confocal microscopy}

HeLa cells were transfected with $2 \mu \mathrm{g}$ of Vpu-EGFP expression plasmids in $10 \mathrm{~cm}$ plates, then 18-24 hours later, seeded on coverslips coated with poly-L-lysine (Sigma Aldrich). The cells were incubated for an 
additional 24 hours at $37^{\circ} \mathrm{C}$, fixed with $4 \%$ paraformaldehyde for 20 minutes at room temperature, washed three times in PBS and subsequently permeabilized for 10 minutes in $0.1 \%$ Triton X-100 at room temperature, followed by washing three times in PBS. Processed cells were mounted in Prolong Gold antifade reagent with DAPI (Molecular Probes, Invitrogen). For analysis of tetherin surface expression, HeLa cells were transfected with $2 \mu \mathrm{g}$ of HIV-1 Vpu or SIVmus/mon/gsn VpuEGFP expression plasmids in $10 \mathrm{~cm}$ plates, then 18-24 hrs later, seeded on coverslips as described above, and incubated at $4^{\circ} \mathrm{C}$ for 20 minutes. Cells were processed for surface tetherin staining by incubation with fresh media plus a polyclonal mouse anti-BST-2 antibody (Abnova, Walnut, CA) at a 1:150 dilution, at $4^{\circ} \mathrm{C}$ for 30 minutes, washed with PBS, fixed with $4 \%$ paraformaldehyde for 20 minutes at room temperature, and washed three times in PBS. To visualize intracellular Vpu proteins, cells were subsequently permeabilized for $10 \mathrm{~min}$ utes in $0.1 \%$ Triton X-100 at room temperature, washed three times in PBS and incubated with a 1:1000 dilution of rabbit anti-Vpu antiserum (ARRRP, from Frank Maldarelli and Klaus Strebel) or a 1:500 dilution of rabbit anti-GFP antibody (Invitrogen). Processed cells were mounted in Prolong Gold antifade reagent with DAPI. Images were acquired using the PerkinElmer Ultraview ERS laser spinning disk confocal imaging system at $100 \times$ magnification (PerkinElmer, Waltham, MA) and processed using Volocity software (Improvision) and Adobe Photoshop Creative Suite 2.

\section{Flow cytometry}

HeLa cells were transfected with an EGFP expression plasmid alone (500 ng), or with $2 \mu \mathrm{g}$ of HIV-1 Vpu or SIVmus/mon/gsn Vpu-EGFP plasmids. Twenty-four hours later, cells were blocked by incubation in $1 \%$ BSA/PBS for 20 minutes at $4^{\circ} \mathrm{C}$ and stained with rabbit anti-BST-2 antiserum (ARRRP, from Klaus Strebel) at a 1:5,000 dilution for 30 minutes at room temperature, followed by washing in PBS and incubation with goat anti-rabbit IgG conjugated to alexa fluor 647 (Invitrogen) at a 1:200 dilution for 20 minutes at room temperature. Cells were analyzed with a FACS CantoII (BD Biosciences, San Jose, CA) and 30,000 events were collected. Data analyses were performed using FlowJo 6.2 software (Tree Star, Ashland, OR).

\section{Acknowledgements}

We thank Beatrice Hahn, Edward Stephens, Jae Jung and Klaus Strebel for generously providing reagents. This study was funded by $\mathrm{NIH}$ grant Al068546 to PMC.

\section{Authors' contributions}

SJY participated in the design of the study, performed most of the experiments, and wrote the draft manuscript. $\mathrm{LAL}, \mathrm{HH}, \mathrm{CME}$ and $\mathrm{KGH}$ contributed to experiments and participated in the review of the manuscript. PMC conceived and coordinated the study, and wrote the final manuscript. All authors read and approved the final manuscript.

\section{Competing interests}

The authors declare that they have no competing interests.

Received: 24 November 2009

Accepted: 18 February 2010 Published: 18 February 2010

References

1. Neil SJD, Sandrin V, Sundquist WI, Bieniasz PD: An Interferon-[alpha]Induced Tethering Mechanism Inhibits HIV-1 and Ebola Virus Particle Release but Is Counteracted by the HIV-1 Vpu Protein. Cell Host \& Microbe 2007, 2:193-203.

2. Neil SJD, Zang T, Bieniasz PD: Tetherin inhibits retrovirus release and is antagonized by HIV-1 Vpu. Nature 2008, 451:425-430.

3. Van Damme N, Goff D, Katsura C, Jorgenson RL, Mitchell R, Johnson MC, Stephens EB, Guatelli J: The Interferon-Induced Protein BST-2 Restricts HIV-1 Release and Is Downregulated from the Cell Surface by the Viral Vpu Protein. Cell Host \& Microbe 2008, 3:245-252.

4. Jouvenet $N$, Neil SJD, Zhadina M, Zang T, Kratovac Z, Lee Y, McNatt M, Hatziioannou T, Bieniasz PD: Broad-Spectrum Inhibition of Retroviral and Filoviral Particle Release by Tetherin. J Virol 2009, 83:1837-1844.

5. Kaletsky RL, Francica JR, Agrawal-Gamse C, Bates P: Tetherin-mediated restriction of filovirus budding is antagonized by the Ebola glycoprotein. Proceedings of the National Academy of Sciences 2009, 106:2886-2891.

6. Sakuma T, Noda T, Urata S, Kawaoka Y, Yasuda J: Inhibition of Lassa and Marburg Virus Production by Tetherin. J Virol 2009, 83:|2382-2385.

7. Abada P, Noble B, Cannon PM: Functional Domains within the Human Immunodeficiency Virus Type 2 Envelope Protein Required To Enhance Virus Production. J Virol 2005, 79:3627-3638.

8. Bour S, Schubert U, Peden K, Strebel K: The envelope glycoprotein of human immunodeficiency virus type 2 enhances viral particle release: a Vpu-like factor?. J Virol 1996, 70:820-829.

9. Jia B, Serra-Moreno R, Neidermyer W Jr, Rahmberg A, Mackey J, Fofana IB, Johnson WE, Westmoreland S, Evans DT: Species-Specific Activity of SIV Nef and HIV-1 Vpu in Overcoming Restriction by Tetherin/BST2. PLOS Pathog 2009, 5:e1000429.

10. Zhang F, Wilson SJ, Landford WC, Virgen B, Gregory D, Johnson MC, Munch J, Kirchhoff F, Bieniasz PD, Hatziioannou T: Nef Proteins from Simian Immunodeficiency Viruses Are Tetherin Antagonists. Cell Host \& Microbe 2009, 6:54-67.

11. Lim ES, Emerman M: Simian Immunodeficiency Virus SIVagm from African Green Monkeys Does Not Antagonize Endogenous Levels of African Green Monkey Tetherin/BST-2. J Virol 2009, 83:11673-11681.

12. Gupta RK, Mlcochova P, Pelchen-Matthews A, Petit SJ, Mattiuzzo G, Pillay D, Takeuchi $Y$, Marsh $M$, Towers GJ: Simian immunodeficiency virus envelope glycoprotein counteracts tetherin/BST-2/CD317 by intracellular sequestration. Proceedings of the National Academy of Sciences 2009.

13. MCNatt MW, Zang T, Hatziioannou T, Bartlett M, Fofana IB, Johnson WE, Neil SJD, Bieniasz PD: Species-Specific Activity of HIV-1 Vpu and Positive Selection of Tetherin Transmembrane Domain Variants. PLoS Pathog 2009, 5:e1000300.

14. Goffinet C, Allespach I, Homann S, Tervo H-M, Habermann A, Rupp D, Oberbremer L, Kern C, Tibroni N, Welsch S, Krijnse-Locker J, Banting G, Kräusslich HG, Fackler OT, Keppler OT: HIV-1 Antagonism of CD317 Is Species Specific and Involves Vpu-Mediated Proteasomal Degradation of the Restriction Factor. Cell Host \& Microbe 2009, 5:285-297.

15. Gupta RK, Hue S, Schaller T, Verschoor E, Pillay D, Towers GJ: Mutation of a Single Residue Renders Human Tetherin Resistant to HIV-1 VpuMediated Depletion. PLoS Pathog 2009, 5:e1000443.

16. Rong L, Zhang J, Lu J, Pan Q, Lorgeoux R-P, Aloysius C, Guo F, Liu S-L, Wainberg MA, Liang C: The Transmembrane Domain of BST-2 Determines Its Sensitivity to Down-Modulation by Human Immunodeficiency Virus Type 1 Vpu. J Virol 2009, 83:7536-7546.

17. Strebel K, Klimkait T, Maldarelli F, Martin MA: Molecular and biochemical analyses of human immunodeficiency virus type 1 vpu protein. $J$ Virol 1989, 63:3784-3791.

18. Schubert U, Bour S, Ferrer-Montiel AV, Montal M, Maldarell F, Strebel K: The two biological activities of human immunodeficiency virus type $1 \mathrm{Vpu}$ 
protein involve two separable structural domains. J Virol 1996, 70:809-819.

19. Dube M, Roy BB, Guiot-Guillain P, Mercier J, Binette J, Leung G, Cohen EA: Suppression of Tetherin-Restricting Activity upon Human Immunodeficiency Virus Type 1 Particle Release Correlates with Localization of Vpu in the trans-Golgi Network. J Virol 2009, 83:4574-4590.

20. Mitchell RS, Katsura C, Skasko MA, Fitzpatrick K, Lau D, Ruiz A, Stephens EB, Margottin-Goguet F, Benarous R, Guatelli JC: Vpu Antagonizes BST-2Mediated Restriction of HIV-1 Release via $\beta$-TrCP and Endo-Lysosomal Trafficking. PLoS Pathog 2009, 5:e1000450.

21. Courgnaud V, Pourrut X, Bibollet-Ruche F, Mpoudi-Ngole E, Bourgeois A, Delaporte E, Peeters M: Characterization of a Novel Simian Immunodeficiency Virus from Guereza Colobus Monkeys (Colobus guereza) in Cameroon: a New Lineage in the Nonhuman Primate Lentivirus Family. J Virol 2001, 75:857-866.

22. Hahn BH, Shaw GM, De KM, Cock, Sharp PM: AIDS as a Zoonosis: Scientific and Public Health Implications. Science 2000, 287:607-614.

23. Huet T, Cheynier R, Meyerhans A, Roelants G, Wain-Hobson S: Genetic organization of a chimpanzee lentivirus related to HIV-1. Nature 1990, 345:356-359.

24. Gao F, Bailes E, Robertson DL, Chen Y, Rodenburg CM, Michael SF, Cummins LB, Arthur LO, Peeters M, Shaw GM, Sharp PM, Hahn BH: Origin of HIV-1 in the chimpanzee Pan troglodytes troglodytes. Nature 1999, 397:436-441

25. Barlow KL, Ajao AO, Clewley JP: Characterization of a Novel Simian Immunodeficiency Virus (SIVmonNG1) Genome Sequence from a Mona Monkey (Cercopithecus mona). J Virol 2003, 77:6879-6888.

26. Courgnaud V, Abela B, Pourrut X, Mpoudi-Ngole E, Loul S, Delaporte E, Peeters M: Identification of a New Simian Immunodeficiency Virus Lineage with a vpu Gene Present among Different Cercopithecus Monkeys (C. mona, C. cephus, and C. nictitans) from Cameroon. J Virol 2003, 77:12523-12534

27. Courgnaud V, Salemi M, Pourrut X, Mpoudi-Ngole E, Abela B, Auzel P, Bibollet-Ruche F, Hahn B, Vandamme A-M, Delaporte E, Peeters M: Characterization of a Novel Simian Immunodeficiency Virus with a vpu Gene from Greater Spot-Nosed Monkeys (Cercopithecus nictitans) Provides New Insights into Simian/Human Immunodeficiency Virus Phylogeny. J Virol 2002, 76:8298-8309.

28. Dazza M-C, Ekwalanga M, Nende M, Shamamba KB, Bitshi P, Paraskevis D, Saragosti S: Characterization of a Novel vpu-Harboring Simian Immunodeficiency Virus from a Dent's Mona Monkey (Cercopithecus mona denti). J Virol 2005, 79:8560-8571.

29. Malim MH, Emerman M: HIV-1 Accessory Proteins-Ensuring Viral Survival in a Hostile Environment. Cell Host \& Microbe 2008, 3:388-398.

30. Sharp PM, Shaw GM, Hahn BH: Simian Immunodeficiency Virus Infection of Chimpanzees. J Virol 2005, 79:3891-3902.

31. Gomez LM, Pacyniak E, Flick M, Hout DR, Gomez ML, Nerrienet E, Ayouba A, Santiago ML, Hahn BH, Stephens EB: Vpu-mediated CD4 down-regulation and degradation is conserved among highly divergent SIVcpz strains. Virology 2005, 335:46-60

32. Pacyniak E, Gomez ML, Gomez LM, Mulcahy ER, Jackson M, Hout DR, Wisdom BJ, Stephens EB: Identification of a Region within the Cytoplasmic Domain of the Subtype B Vpu Protein of Human Immunodeficiency Virus Type 1 (HIV-1) That Is Responsible for Retention in the Golgi Complex and Its Absence in the Vpu Protein from a Subtype C HIV-1. AIDS Research and Human Retroviruses 2005, 21:379-394.

33. Douglas JL, Viswanathan K, McCarroll MN, Gustin JK, Fruh K, Moses AV: Vpu Directs the Degradation of the Human Immunodeficiency Virus Restriction Factor BST-2/Tetherin via a \{beta\}TrCP-Dependent Mechanism. J Virol 2009, 83:7931-7947.

34. Takehisa J, Kraus MH, Ayouba A, Bailes E, Van Heuverswyn F, Decker JM, Li Y, Rudicell RS, Learn GH, Neel C, Ngole EM, Shaw GM, Peeters M, Sharp PM, Hahn BH: Origin and Biology of Simian Immunodeficiency Virus in Wild-Living Western Gorillas. J Virol 2009, 83:1635-1648.

35. Wain LV, Bailes E, Bibollet-Ruche F, Decker JM, Keele BF, Van Heuverswyn F, Li Y, Takehisa J, Ngole EM, Shaw GM, Peeters M, Hahn BH, Sharp PM: Adaptation of HIV-1 to Its Human Host. Mol Biol Evol 2007, 24:1853-1860.

36. Sauter D, Schindler M, Specht A, Landford WN, Münch J, Kim K-A, Votteler J, Schubert U, Bibollet-Ruche F, Keele BF, Takehisa J, Ogando Y, Ochsenbauer C, Kappes JC, Ayouba A, Peeters M, Learn GH, Shaw G, Sharp PM, Bieniasz P, Hahn BH, Hatziioannou T, Kirchhoff F: Tetherin-Driven
Adaptation of Vpu and Nef Function and the Evolution of Pandemic and Nonpandemic HIV-1 Strains. Cell Host Microbe 2009, 6:409-421.

37. Miyagi $E$, Andrew AJ, Kao S, Strebel K: Vpu enhances HIV-1 virus release in the absence of Bst-2 cell surface down-modulation and intracellular depletion. Proceedings of the National Academy of Sciences 2009, 106:2868-2873

38. Klimkait T, Strebel K, Hoggan MD, Martin MA, Orenstein JM: The human immunodeficiency virus type 1-specific protein vpu is required for efficient virus maturation and release. J Virol 1990, 64:621-629.

39. Ritter GD Jr, Yamshchikov G, Cohen SJ, Mulligan MJ: Human immunodeficiency virus type 2 glycoprotein enhancement of particle budding: role of the cytoplasmic domain. J Virol 1996, 70:2669-2673.

40. Schindler M, Münch J, Kutsch O, Li H, Santiago ML, Bibollet-Ruche F, MüllerTrutwin MC, Novembre FJ, Peeters M, Courgnaud V, Bailes E, Roques P, Sodora DL, Silvestri G, Sharp PM, Hahn BH, Kirchhoff F: Nef-Mediated Suppression of T Cell Activation Was Lost in a Lentiviral Lineage that Gave Rise to HIV-1. Cell 2006, 125:1055-1067.

41. Kirchhoff F: Is the high virulence of HIV-1 an unfortunate coincidence of primate lentiviral evolution?. Nat Rev Micro 2009, 7:467-476.

42. Nguyen K-L, Llano M, Akari H, Miyagi E, Poeschla EM, Strebel K, Bour S: Codon optimization of the HIV-1 vpu and vif genes stabilizes their mRNA and allows for highly efficient Rev-independent expression. Virology 2004, 319:163-175.

43. Noble B, Abada P, Nunez-Iglesias J, Cannon PM: Recruitment of the Adaptor Protein 2 Complex by the Human Immunodeficiency Virus Type 2 Envelope Protein Is Necessary for High Levels of Virus Release. J Virol 2006, 80:2924-2932.

44. Müller-Trutwin MC, S C, Souquière $S$, Roques $P$, Versmisse $P$, Ayouba $A$, Delarue S, Nerrienet E, Lewis J, Martin P, Simon F, Barré-Sinoussi F, Mauclère P: SIVcpz from a naturally infected Cameroonian chimpanzee: biological and genetic comparison with HIV-1 N. J Med Primatology 2000, 29:166-172.

45. Hirsch VM, Olmsted RA, Murphey-Corb M, Purcell RH, Johnson PR: An African primate lentivirus (SIVsm) closely related to HIV-2. Nature 1989, 339:389-392.

46. Novembre FJ, Hirsch VM, McClure HM, Fultz PN, Johnson PR: SIV from stump-tailed macaques: Molecular characterization of a highly transmissible primate lentivirus. Virology 1992, 186:783-787.

47. Müller MC, Barré-Sinoussi F: SIVagm: genetic and biological features associated with replication. Front Biosci 2003, 8:d1170-1185.

48. Hirsch VM, Dapolito GA, Goldstein S, McClure H, Emau P, Fultz PN, Isahakia M, Lenroot R, Myers G, Johnson PR: A distinct African lentivirus from Sykes' monkeys. J Virol 1993, 67:1517-1528.

49. Hirsch VM, Campbell BJ, Bailes E, Goeken R, Brown C, Elkins WR, Axthelm M, Murphey-Corb M, Sharp PM: Characterization of a Novel Simian Immunodeficiency Virus (SIV) from L'Hoest Monkeys (Cercopithecus I'hoesti): Implications for the Origins of SIVmnd and Other Primate Lentiviruses. J Virol 1999, 73:1036-1045.

50. Le Tortorec A, Neil SJD: Antagonism to and Intracellular Sequestration of Human Tetherin by the Human Immunodeficiency Virus Type 2 Envelope Glycoprotein. J Virol 2009, 83:11966-11978.

doi:10.1186/1742-4690-7-13

Cite this article as: Yang et al:: Anti-tetherin activities in Vpu-expressing primate lentiviruses. Retrovirology 2010 7:13.

\section{Submit your next manuscript to BioMed Central and take full advantage of:}

- Convenient online submission

- Thorough peer review

- No space constraints or color figure charges

- Immediate publication on acceptance

- Inclusion in PubMed, CAS, Scopus and Google Scholar

- Research which is freely available for redistribution 